\title{
Accelerating implementation of biomedical research advances: critical elements of a successful 10 year Cystic Fibrosis Foundation healthcare delivery improvement initiative
}

\author{
Bruce C Marshall, ${ }^{1}$ Eugene C Nelson ${ }^{2}$
}

${ }^{1}$ Cystic Fibrosis Foundation, Bethesda, Maryland, USA ${ }^{2}$ Dartmouth-Hitchcock Medical Center and The Dartmouth Institute for Health Policy and Clinical Practice, Lebanon, New Hampshire, USA

Correspondence to Dr B C Marshall, Cystic Fibrosis Foundation, 6931 Arlington Road, Bethesda, MD 20814, USA; bmarshall@cff.org

Received 30 December 2013 Accepted 6 January 2014
CrossMark

To cite: Marshall $B C$ Nelson EC. BMJ Qual Saf 2014:23:i95-i103.

\begin{abstract} Context: Scientific and therapeutic advances Remarkable biomedical research advances have led to innovative and increasingly effective therapies. We highlight several scientific milestones in elucidating the pathophysiology of cystic fibrosis (CF) and review the therapies that have become available over the past 20 years. Impact of the quality improvement initiative In 2002, the CF Foundation launched a multifaceted quality improvement initiative to accelerate improvement in CF care. We present evidence of substantial improvement in process measures, such as more consistent outpatient follow-up, and key medical outcomes, including survival, pulmonary function and nutritional status. Critical success factors We offer our perspective on factors critical to the success of the quality improvement initiative, including a compelling strategic plan and the commitment of the CF Foundation to its implementation; the investment in building improvement capacity at CF care centres; the engagement of people with CF and their families as partners; and the integration of quality improvement into the existing CF care framework
\end{abstract}

Directions for the next decade In addition to a continued investment in building and sustaining improvement capacity at CF care centres, and deeper patient engagement, we will address the oppressive treatment burden. We will also complement the measurement of clinical outcomes with patient reported outcomes and healthcare costs for a balanced assessment of the quality and value of care.

Conclusions Major advances in basic science and therapeutic development coupled with improvements in healthcare delivery have resulted in striking gains in medical outcomes for people with CF.

\section{INTRODUCTION}

The cystic fibrosis (CF) community has benefited from remarkable biomedical research advances, which have led to innovative and increasingly effective therapies. We propose that this success has been magnified and accelerated by the introduction of healthcare improvement strategies into the CF care model. Improved process measures including more consistent outpatient follow-up, better vaccination rates, more systematic screening for co-morbidities and wider use of proven therapies have been associated with improvements in key clinical outcomes such as survival, pulmonary function and nutritional status. We offer our perspective on the factors critical to the success of the quality improvement initiative and close with our thoughts on directions for the upcoming decade.

\section{CONTEXT: SCIENTIFIC AND THERAPEUTIC ADVANCES}

Unravelling the pathophysiology

At the time of Dr Dorothy Andersen's (figure 1) early description of $\mathrm{CF}$ in $1938,{ }^{1}$ the prognosis was dismal and the underlying pathogenesis was poorly understood. An astute clinical observation of children with CF presenting with heat prostration during a torrid New York City heat wave in August $1948^{2}$ provided a clue to the pathogenesis. The discovery of strikingly elevated chloride and sodium levels in the sweat of individuals with $\mathrm{CF}$ by Paul di Sant'Agnese (figure 2) and colleagues explained the increased risk of dehydration ${ }^{3}$ and eventually led to the diagnostic sweat chloride test. ${ }^{4}$ Further 


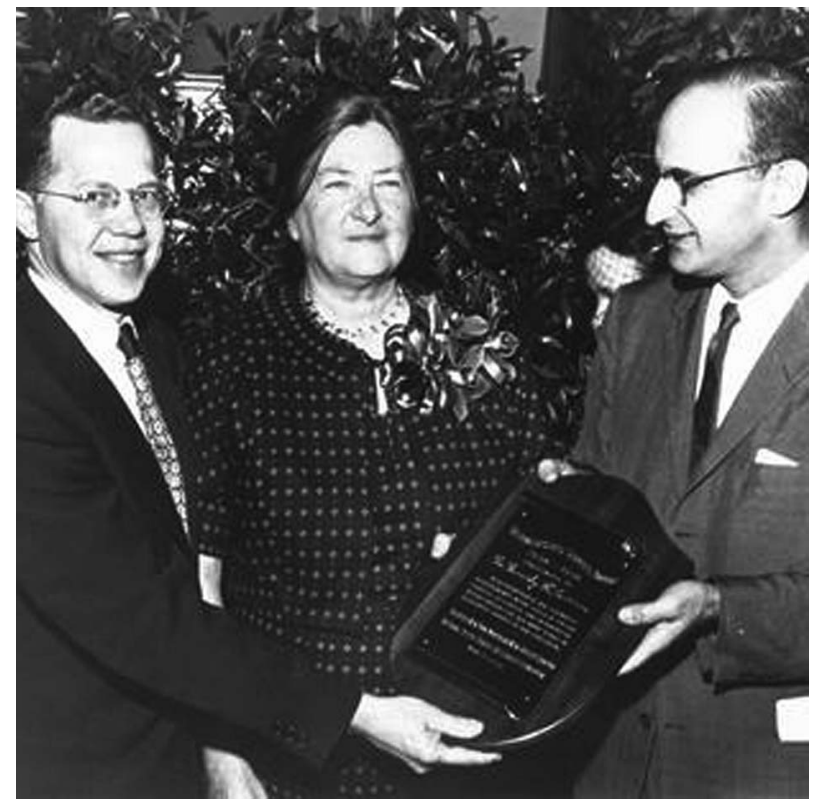

Figure 1 Dr Dorothy $\mathrm{H}$ Andersen, accepting an award for her early description of cystic fibrosis, from Robert Natal (right), president of the New York Chapter of the National Cystic Fibrosis Foundation, and Victor Blitzer, former president, 1958. Library of Congress, Prints and Photographs Division, New York World Telegram and Sun Collection.

research showing chloride impermeability in the sweat duct $^{5}$ and similar abnormalities in the airway epithelium ${ }^{6}$ linked the scientific findings to the clinical manifestations of the disease (see box 1).

The discovery of the CF transmembrane conductance regulator (CFTR) gene and the major mutation (F508del) in 1989 by Drs Lap-Chee Tsui, Jack Riordan and Francis Collins (figure 3) and colleagues ${ }^{7}$ was a pivotal milestone in unravelling the pathophysiology of this autosomal recessive disease. The CFTR protein proved to be a regulated membrane anion channel, ${ }^{8}$ thus connecting the gene, protein and cellular abnormalities. Hope that this breakthrough would

Box 1 Unravelling the pathophysiology: scientific milestones

- 1938: Early description of the disease as distinct from coeliac disease

- 1948: Clinical presentation of cystic fibrosis patients with heat prostration

1953: Discovery of elevated sweat chloride

- 1963: Development of the diagnostic sweat chloride test

- 1983: Discovery of chloride impermeability in sweat ducts and airway epithelium

- 1989: Discovery of the cystic fibrosis transmembrane conductance regulator gene and major mutation (F508del)

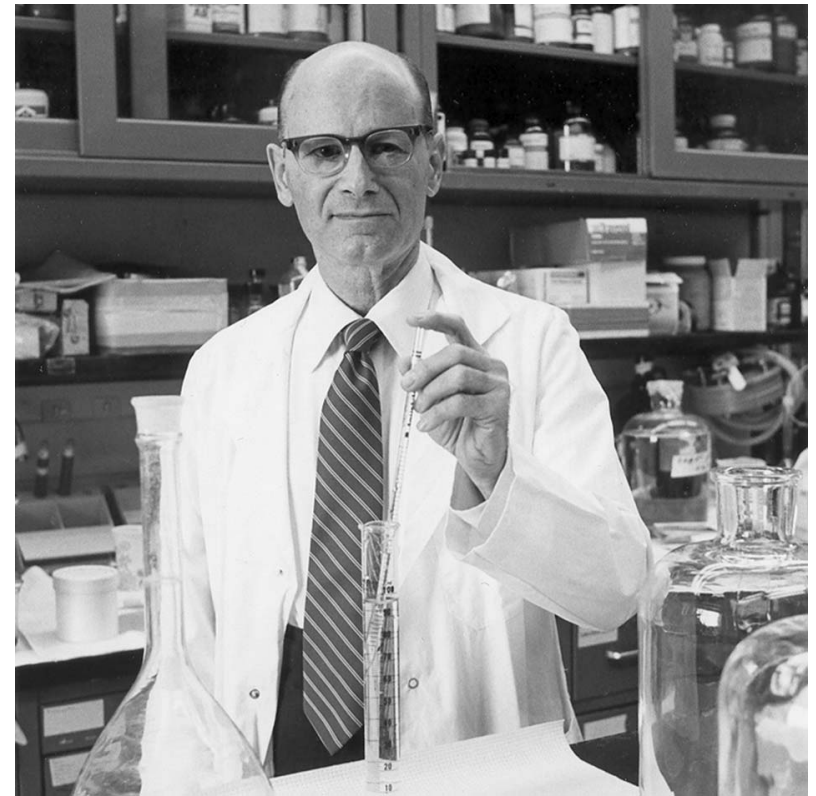

Figure 2 Dr Paul di Sant'Agnese in his laboratory in 1953

lead to a gene transfer cure faded with the daunting challenge of efficiently delivering the normal CFTR gene to airway cells without inducing inflammation. ${ }^{9}$

\section{Advances in pulmonary therapeutics}

As the basic biology of the CFTR gene and protein advanced, the CF community aggressively pursued the development of therapies targeting the pulmonary manifestations of the disease, the major cause of mortality. Several therapies were shown to improve pulmonary function and/or decrease pulmonary

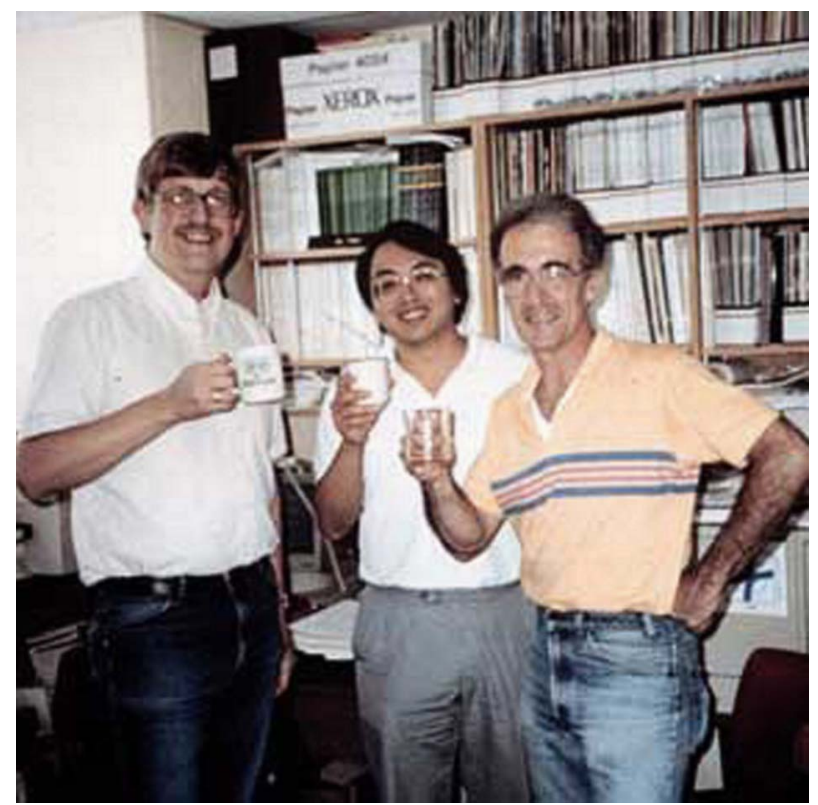

Figure 3 Drs Francis Collins, Lap-Chee Tsui and Jack Riordan (left to right) celebrate their 1989 discovery of the cystic fibrosis gene. 


\section{Box 2 Advances in pulmonary therapeutics}

1993: Dornase alfa, US regulatory approval

- 1997: Tobramycin solution for inhalation, US regulatory approval

- 2003: Azithromycin, published randomised controlled trial showing safety and effectiveness

- 2006: Hypertonic saline, published randomised controlled trial showing safety and effectiveness

- 2010: Aztreonam solution for inhalation, US regulatory approval

exacerbations (see box 2). Inhaled dornase $\mathrm{alfa}^{10}$ and tobramycin solution for inhalation ${ }^{11}$ received US regulatory approval in 1993 and 1997, respectively. Azithromycin for patients chronically infected with Pseudomonas aeruginosa ${ }^{12}$ and inhaled hypertonic saline ${ }^{13}$ were reported to be effective in clinical trials in 2003 and 2006, respectively. Another inhaled antibiotic, aztreonam for inhalation solution, ${ }^{14}{ }^{15}$ received US regulatory approval in 2010. These chronic pulmonary therapies are recommended in evidence based clinical practice guidelines ${ }^{16}{ }^{17}$ and have been widely adopted at CF care centres.

\section{Targeting the basic defect}

While these therapeutic advances were laudable, drugs targeting the basic defect were notably lacking. To address this void, the CF Foundation launched the Therapeutics Development Programme (TDP) in 1998 to attract the expertise and resources of pharmaceutical and biotechnology companies by lowering their risk of entering $\mathrm{CF}$ research. In addition to financial support of TDP awardees via milestone driven grants, the Foundation contributes clinical and scientific knowledge of the disease, and access to the CF care centre and clinical research networks. Success, as evidenced by regulatory approval of a drug, triggers a financial return to the Foundation to support CF research and clinical programmes. Other voluntary health care organisations have emulated this innovative 'venture philanthropy' model ${ }^{18}$ created by Dr Robert J Beall (figure 4).

The first TDP collaboration, formed with Aurora Biosciences (subsequently acquired by Vertex Pharmaceuticals) in 1999, funded high throughput screening of chemical libraries in search of CFTR modulators (correctors and potentiators). Correctors target the misfolding of CFTR protein which disrupts transport to the cell membrane, and potentiators target the defective gating of CFTR protein at the cell membrane. Ivacaftor, the initial output from this collaboration, is a potentiator that targets the gating defect caused by the G551D mutation present in approximately $4 \%$ of individuals with CF in the USA.

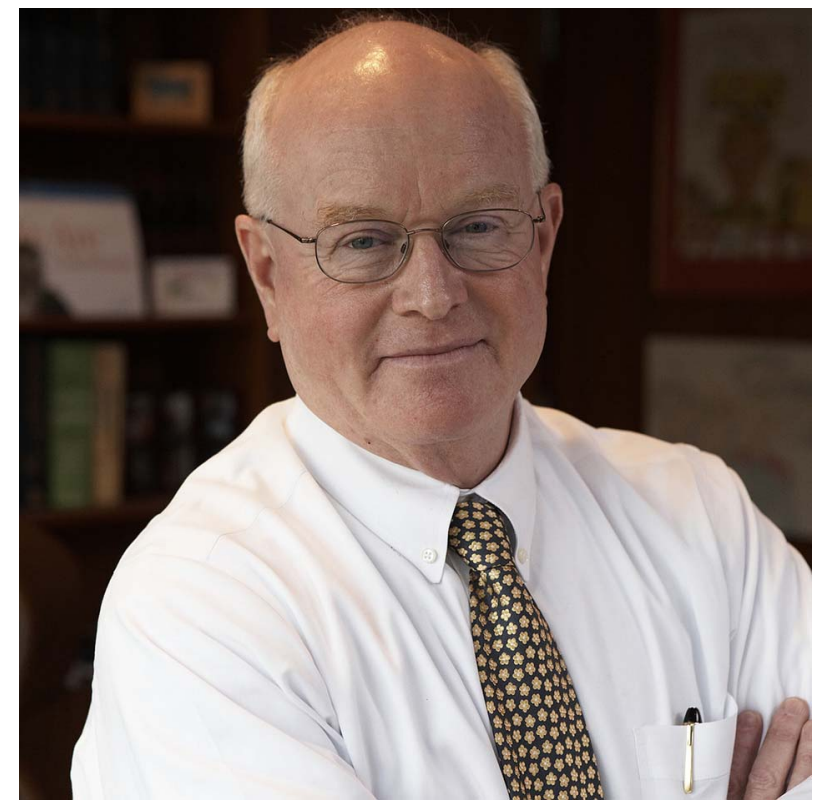

Figure 4 Dr Robert J Beall, President and CEO of the Cystic Fibrosis Foundation, creator of the Therapeutics Development Programme.

Clinical trials of ivacaftor showed marked improvement in medical outcomes as well as a striking decrease in sweat chloride values to below the standard diagnostic cut-off for $\mathrm{CF},{ }^{19}$ confirming CFTR as the drug target. The strength of these findings led to rapid US regulatory approval in 2012. Ivacaftor is now widely prescribed for eligible patients, and evidence suggests that it is highly effective in the clinical setting. ${ }^{20}$ The development path for ivacaftor provides a roadmap for other potential CFTR modulators, which could change the face of the disease.

\section{IMPACT OF THE QUALITY IMPROVEMENT INITIATIVE}

Improvements in CF care delivery and medical outcomes kept pace with the remarkable scientific and therapeutic advances. In 2002, the CF Foundation launched a multifaceted quality improvement initiative to accelerate improvement in CF care. ${ }^{21}$ The cornerstones of the initiative are described in detail elsewhere in this supplement and include leadership development, scientific approach to change, patient engagement and implementation of clinical practice guidelines. The data shown below are derived from CF Foundation's patient registry, ${ }^{22}$ which serves as a rich resource to evaluate practice patterns and medical outcomes over time.

\section{Process measures}

A number of care process measures derived from guideline recommendations have shown improvement over the past decade. Quarterly follow-up is recommended for close monitoring of signs and symptoms, respiratory microbiology, pulmonary function and 
nutritional status. The proportion of children 6-17 years of age with four or more clinic visits, four or more respiratory cultures and two or more pulmonary function tests increased from $25 \%$ in 2003 to $57 \%$ in 2012. Among children with suboptimal nutritional status (body mass index (BMI) $<50$ th percentile for age), the per cent evaluated by a dietitian, increased from $78 \%$ in 2003 to $94 \%$ in 2012.

Regular clinical follow-up also provides opportunities for preventive interventions and screening for comorbidities. The influenza vaccination rate for individuals with $\mathrm{CF} \geq 6$ months of age increased from $88 \%$ in 2006 (27.8\% of patients with unknown vaccination status excluded from the analysis) to $94 \%$ in $2012(16.3 \%$ of patients with unknown vaccination status excluded from the analysis). From 2002 to 2012, the prevalence of depression and osteoporosis for individuals with $\mathrm{CF} \geq 18$ years of age increased from $10.4 \%$ to $22.2 \%$ and from $3.7 \%$ to $8.5 \%$, respectively, likely due to more systematic screening. Although the improvements in process measures are encouraging, the causal link to clinical outcomes cannot be clearly established absent a prospective trial.

\section{Survival}

The success of the CF care model is evident in the dramatic improvement in survival from the 1960s when most children with CF did not reach school age. ${ }^{23} 24$ Accreditation of care centres across the USA in the 1960 s, 1970s and 1980s, and the associated standardisation of multidisciplinary care, certainly contributed to the improved survival. Survival has continued to improve, most notably over the past decade. Median predicted survival, calculated by life table analysis, increased from 29.4 (95\% CI 28.4 to 30.8) years in 1992, to 31.3 (95\% CI 29.7 to 33.1 ) years in 2002, and to 41.1 (95\% CI 37.4 to 43.1) years in 2012. Nearly half of the CF patient population in the USA is now 18 years of age or older.

\section{Pulmonary function}

Pulmonary function has also markedly improved (figure 5). A sceptic might argue that the pulmonary function improvement was simply due to the introduction of the chronic pulmonary therapies into clinical practice. However, we believe that the therapies and the quality improvement initiative were complementary drivers of improved outcomes. New therapies are often slow to diffuse into routine practice without a promotional campaign and/or strategies to facilitate implementation. A number of CF care centres focused their improvement work on ensuring that the pulmonary therapies were prescribed for eligible patients. ${ }^{25}$ This likely contributed to the clinical adoption of the pulmonary therapies, particularly azithromycin and hypertonic saline (figure 6), which do not have regulatory approval for their use in CF and are therefore not promoted to the CF care centres by pharmaceutical companies.

\section{Median FEV $\mathbf{1}_{1}$ Percent Predicted by Age}

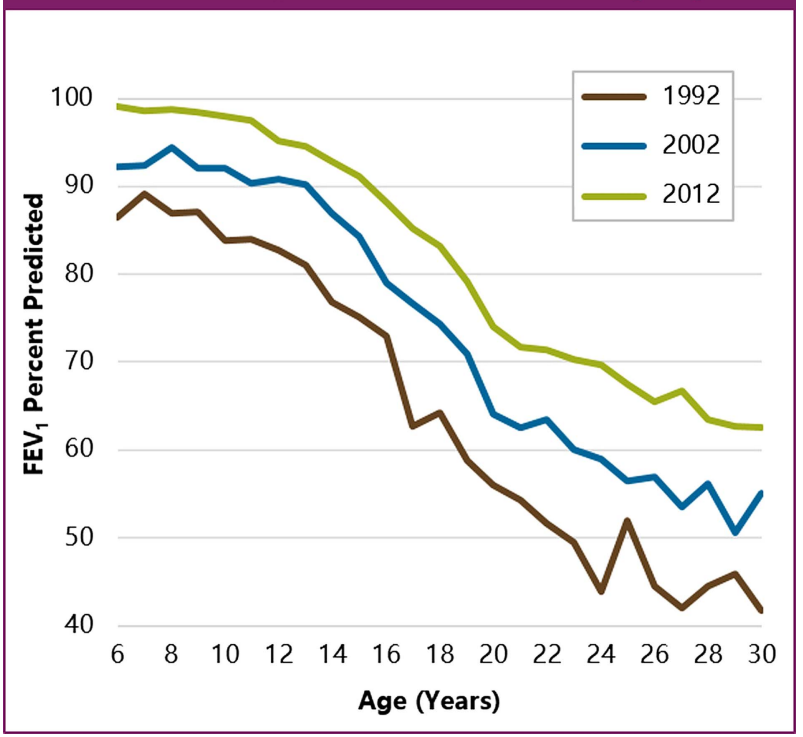

Figure 5 Cross sectional analyses of registry data from 1992 to 2012 show improved forced expiratory volume in $1 \mathrm{~s}\left(\mathrm{FEV}_{1}\right)$ (\% predicted).

\section{Nutritional outcomes}

Many CF care centres focused on improving the nutritional status of their patient population, outcomes which are much less likely to be confounded by the introduction of the pulmonary therapies. In addition to the improvement in BMI percentile for

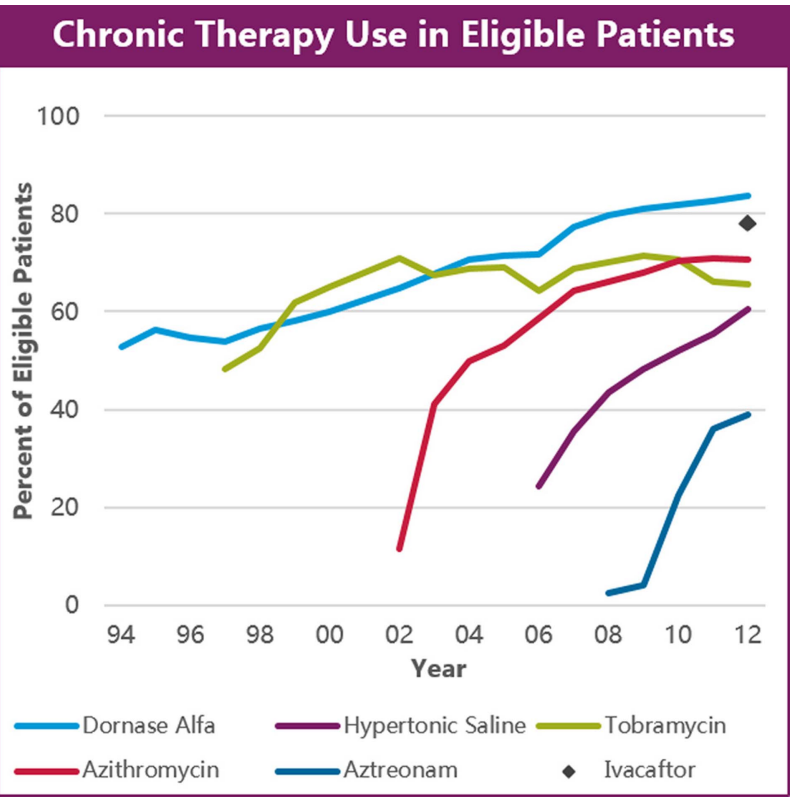

Figure 6 The use of therapies in eligible patients from registry data is shown from 1994 to 2012. Explicit criteria for eligibility were developed from recommendations in published clinical practice guidelines. ${ }^{16} 17$ Patients are included if they were prescribed the specific therapy at any encounter during the year. 


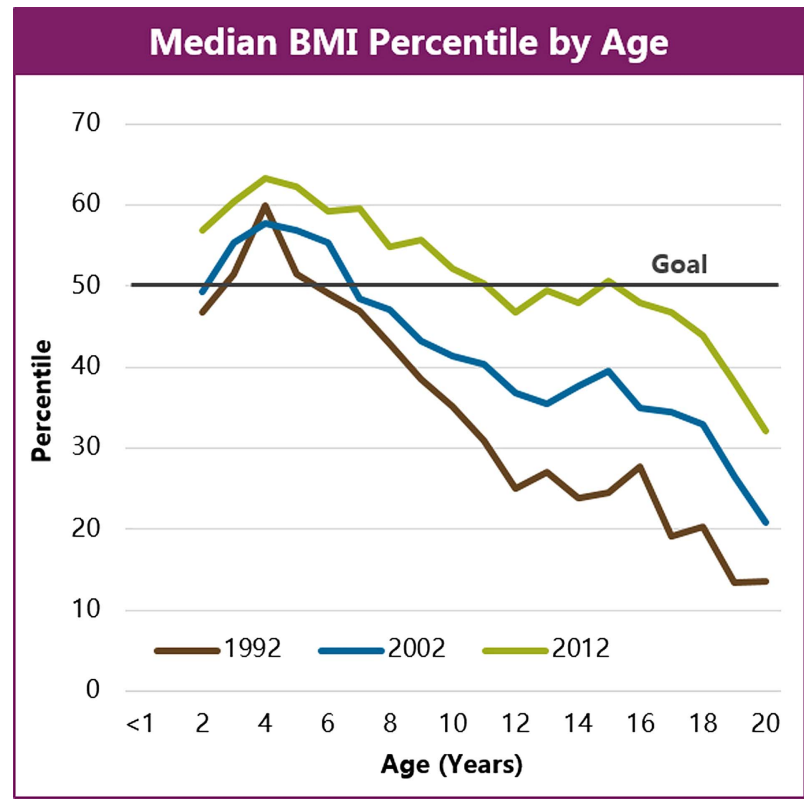

Figure 7 Cross sectional analyses of registry data from 1992 to 2012 show improved body mass index (BMI) percentiles. Calculated from 2000 US Center for Disease Control and Prevention reference values. ${ }^{25 a}$

children (figure 7), remarkable improvement in height and weight for age percentiles has occurred over the past decade compared with previous decades (figures 8,9). Newborn screening for CF, implemented in the great majority of states in the mid to late 2000s, may have contributed to the improved nutritional measures in younger children, but not for the

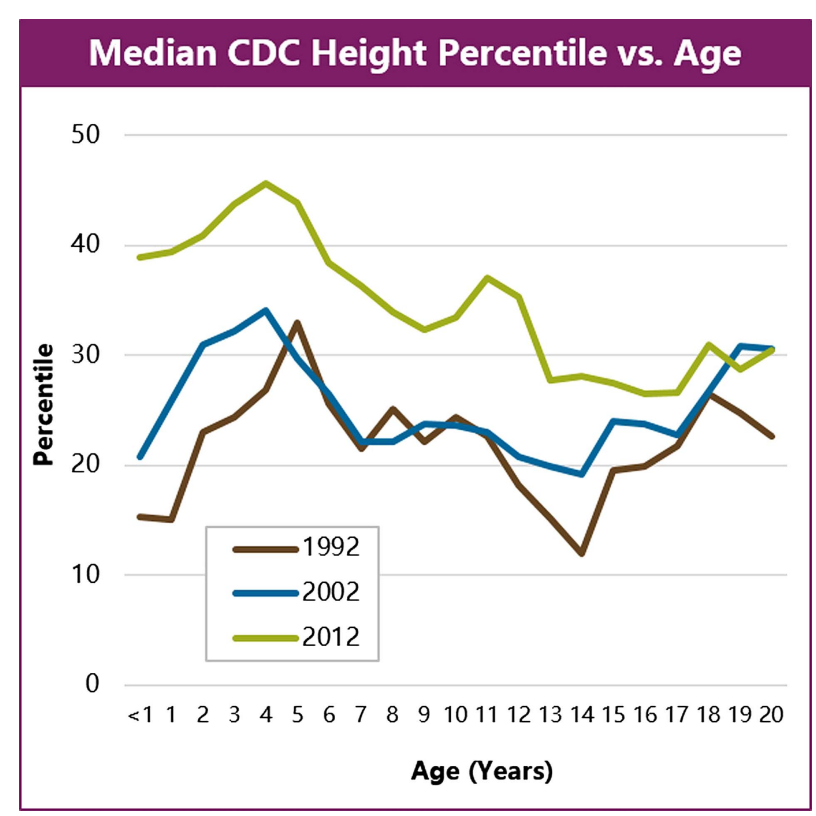

Figure 8 Cross sectional analyses of registry data from 1992 to 2012 show improved height for age percentiles. The 2012 data show what appears to be a pubertal growth spurt, not evident in the 1992 or 2002 data calculated from 2000 US Center for Disease Control and Prevention reference values. ${ }^{25 a}$ older children and adolescents. These data suggest that the quality improvement initiative contributed to the impressive gains in nutritional outcomes over the past decade.

\section{Centre level outcomes}

The impact of quality improvement is most apparent in the centre level data. Figure 10 shows pulmonary and nutritional outcomes for children with CF. Each dot represents the median values for a CF care centre. The magnitude of the overall change in median values from 2002 to 2012 (forced expiratory volume in $1 \mathrm{~s}$ $\left(\mathrm{FEV}_{1}\right)$ per cent predicted +6.0 ; $\mathrm{BMI}$ percentile +10.5$)$ is clinically significant. By $2012, \mathrm{FEV}_{1}$ per cent predicted for many centres approached or exceeded 100\%, diminishing the potential for further improvement (ie, 'ceiling effect'). Many, but not all, centres that engaged in and sustained quality improvement activities have shown significant improvement in pulmonary and/or nutritional outcomes. For example, the CF care centres reporting their work in this supplement have achieved substantial gains, particularly in nutritional outcomes (see table 1).

In summary, given the complexity of the healthcare system and the introduction of new therapies, a causal link between quality improvement and improved national level outcomes cannot be proven. However, the circumstantial evidence suggests a substantial impact and supports the value of this approach for other chronic diseases.

\section{CRITICAL SUCCESS FACTORS}

We identified five factors critical to the success of the quality improvement initiative (box 3) supported by

\section{Median CDC Weight Percentile vs. Age}

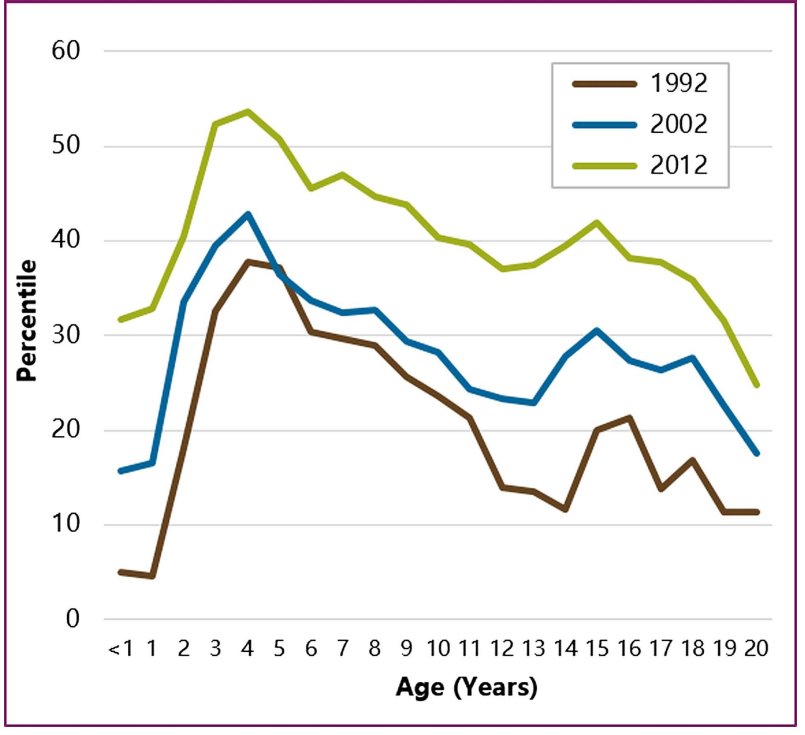

Figure 9 Cross sectional analyses of registry data from 1992 to 2012 show improved weight for age percentiles. Calculated from 2000 US Center for Disease Control and Prevention reference values. ${ }^{25 a}$ 


\section{FEV 1 vs. BMI Percentile for Patients 6 to 17 Years}

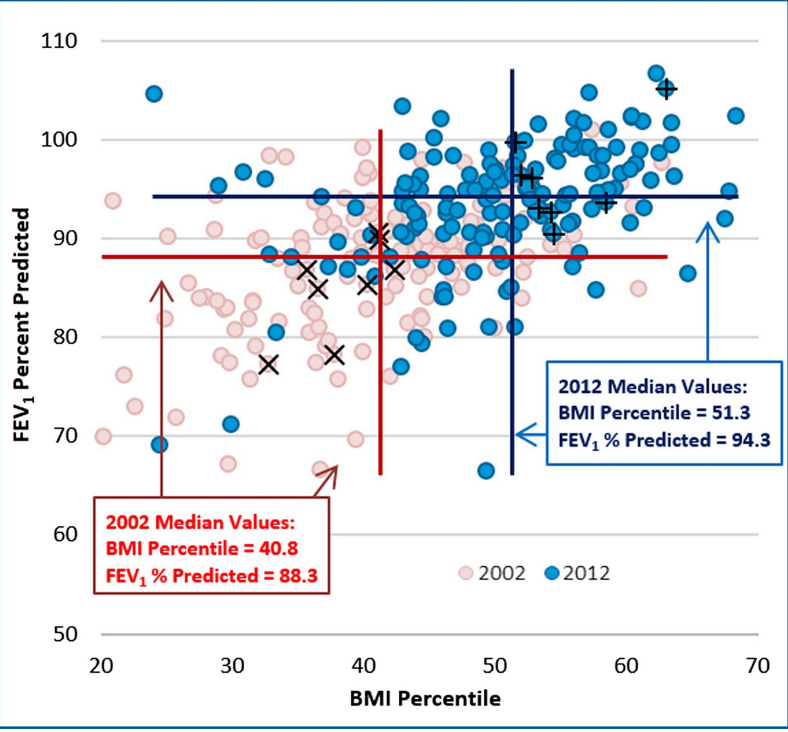

Figure 10 Forced expiratory volume in $1 \mathrm{~s}\left(\mathrm{FEV}_{1}\right)$ versus body mass index (BMI) percentiles for patients aged 6 to 17 years. Each pink dot represents the median values for BMI percentile and $\mathrm{FEV}_{1}$ per cent predicted for children at an individual care centre in 2002, and each blue dot represents the median values in 2012. The red and black lines show the overall population medians for BMI percentile and FEV 1 per cent predicted for 2002 and 2012, respectively. The dots with hatched marks $(x-2002$ and + -2012) show the care centres reporting their improvement activities in this supplement. Their improvement in outcomes compared with all other care centres is shown in table 1.

the strategic investment of the CF Foundation. The multidisciplinary CF care model with peer review and a long standing patient registry ${ }^{21}{ }^{26}$ provided fertile ground for this work. The importance of these existing assets should not be overlooked. The strategic plan coupled with the commitment of the CF Foundation to its implementation led to an investment in building improvement capacity at CF care centres and the engagement of people with CF and their families as partners in the improvement work.

Table 1 Improvement in pulmonary and nutritional outcomes from 2002 to 2012

Mean difference between 2002 and 2012

\begin{tabular}{llll}
\cline { 2 - 4 } & $\begin{array}{l}\text { Centres in } \\
\text { supplement† }\end{array}$ & $\begin{array}{l}\text { All other } \\
\text { centres }\end{array}$ & p Value* \\
\hline BMI percentile & 16.7 & 10.0 & 0.005 \\
$\mathrm{FEV}_{1}(\%$ & 10.9 & 5.9 & 0.07 \\
predicted) & & & \\
\hline
\end{tabular}

*t test.

†Med College of Wisconsin, Children's Hospital of Atlanta, Arkansas Children's Hospital, Monmouth Medical Center, Cincinnati Children's Medical Center, University of California, San Francisco, Lurie Children's Hospital of Chicago, Children's of Alabama.

$\mathrm{BMI}$, body mass index; FEV 1 , forced expiratory volume in $1 \mathrm{~s}$.

\section{Box 3 Critical success factors}

1. Compelling strategic plan (ie, opportunity statement)

2. Commitment of the Cystic Fibrosis Foundation to implementation

3. Investment in building improvement capacity at cystic fibrosis care centres

4. Focus on engaging people with cystic fibrosis and their families

5. Integration of quality improvement into the cystic fibrosis care framework

Factors 1 and 2: compelling strategic plan and commitment to implementation

Acknowledging the wide variation in outcomes across the care centre network and engaging external improvement experts in the strategic planning process took courage on the part of CF Foundation leaders. The Opportunity Statement that resulted from the strategic planning process stated the vision of exemplary care at all CF care centres and created a sense of urgency for improvement. Key strategies and worthy goals provided a focus for improvement activities. ${ }^{21}$ Credible clinical leadership at the CF Foundation coupled with improvement expertise at Dartmouth ensured high visibility of the initiative. Learning and Leadership Collaboratives were organised (see below) and a Quality Improvement Grants Programme was established to support innovative work. Progress was regularly featured at the Annual North American CF Conference, leaving no doubt about the CF Foundation's commitment.

\section{Factor 3: investment in building improvement capacity at CF care centres}

Godfrey $e t a l^{27}$ describe the key elements of the year long CF Foundation sponsored Learning and Leadership Collaboratives, an important mechanism for engaging $\mathrm{CF}$ care centres in quality improvement. We realised that reaching over 110 accredited care centres (comprised of over 250 distinct paediatric, adult and affiliate programmes) would take considerable time and resources. We initially focused on recruiting clinical leaders in the community and searched for early success stories that could be shared peer to peer. The development of coaches to support the teams participating in the collaboratives allowed us to reach more care centres faster. By furthering the knowledge and experience of the coaches, we garnered strong advocates for quality improvement and enhanced the sustainability of improvement at their centres.

Benchmarking visits to high performing centres demonstrated the importance of factors other than the technical delivery of care. Optimal outcomes at $\mathrm{CF}$ care centres were associated with effective 
leadership and team dynamics, close contact with patients and families, high expectations, and a proactive and consistent practice approach. ${ }^{28}$ These findings validated our focus on developing the improvement capabilities of frontline CF care teams.

The major impact of the unexpected departure of an experienced CF dietitian was an incidental finding at one of the benchmarking visits. This observation led to the creation of mentoring programmes to pair an apprentice new to CF care with an experienced mentor in the same discipline from another care centre. Individual goals with a focus on improving care processes and outcomes are established for each apprentice prior to a site visit to the mentor's CF care centre. These highly successful programmes, ${ }^{29}$ now operational in several disciplines, foster the development of communities of practice ${ }^{30}$ and facilitate cross pollination of ideas for improvement.

\section{Factor 4: focus on engaging people with CF and their families}

The perspective and practical knowledge of individuals with CF and/or parents on the teams that participated in a Learning and Leadership Collaborative enriched the improvement work and motivated the clinicians. Patient/family advisory boards were established at many CF care centres to gain broader input on improvement opportunities. Individuals with $\mathrm{CF}$ and/or parents were also invited to serve on clinical practice guidelines committees, contributing to development of recommendations for care and complementing local efforts to improve care at their own $\mathrm{CF}$ centres. $^{31}$

We recognised that achieving optimal outcomes required a strong partnership between people with CF and healthcare professionals. People with CF and their families consent to the collection of their data for the patient registry, so they, as partners, should have access to the centre level outcome measures. Schechter et $a^{26}$ describe our path to data transparency and the public report of outcomes and process measures on the CF Foundation website (http://www. cff.org). To put it simply, it became clear that it was the right thing to do and it would likely help us accelerate improvement.

\section{Factor 5: integration of quality improvement into the CF care framework}

The integration of quality improvement into the existing CF care framework ensured sustainability. The members of the Centre Committee broadened their mission statement to encompass 'fostering exemplary care' as well as ensuring that centres met accreditation standards. A report on quality improvement activities was required in annual progress reports from care centres and at accreditation site visits. Quality award winners were selected by the Centre Committee and publicly recognised at the North American CF
Conference. Participation in quality improvement has now become an expectation, driven more by the $\mathrm{CF}$ community than by central leadership.

\section{DIRECTIONS FOR THE UPCOMING DECADE}

Substantial progress has been made since Andersen's description of CF, but much work remains. While the improvement in clinical outcomes is remarkable, life expectancy is still decades below that of the general population. This highlights the need for additional CFTR modulators, and the CF Foundation is investing significant resources in facilitating the development of these drugs. We are both realistic and optimistic about the challenges ahead. To ensure that people with CF fully benefit from these potential disease modifying therapies, we remain committed to the vision of exemplary care for all people with CF.

Directions for the upcoming decade are listed in box 4 . We remain firmly committed to an ongoing investment in building and sustaining improvement capacity at CF care centres and deeper patient engagement. We will also address a hidden cost of improved outcomes-that is, the oppressive treatment burden for individuals with CF-by: (1) gaining deeper knowledge of the challenges that individuals with $\mathrm{CF}$ and families face in adhering to the recommended treatment regimen and (2) applying comparative effectiveness research methodologies to the registry data. Finally, we plan to complement the registry data with patient reported outcomes and cost outcomes for a broader and more balanced assessment of the quality and value of CF care.

\section{CF care centres as patient centred medical homes}

We continue to support the development of the CF care centre teams by providing opportunities to participate in improvement collaboratives, leadership development and mentoring programmes. Building on the success of improvement work within CF care centre microsystems, we are also focusing on improving interactions with other related microsystems. ${ }^{32}$ For example, CF related diabetes is a highly prevalent comorbidity $^{33}$ associated with increased mortality and increased treatment burden. The care of an individual

\section{Box 4 Directions for the upcoming decade}

Develop cystic fibrosis care centres as patient centred medical homes

- Deeper patient engagement

- Address the treatment burden

- Comparative effectiveness research

- Barriers to adherence

- Develop metrics to provide a balanced assessment of quality and value of care 
with CF related diabetes requires coordination between the CF care centre microsystem and an endocrinologist and care team in another microsystem. A well thought out referral plan, communication plan and role definition for the two interdependent microsystems are important to achieve optimal outcomes.

We aspire to seamless integration within CF care centres (ie, between paediatric and adult CF programmes, and between inpatient and outpatient units) and with interfacing subspecialists and programmes (eg, state newborn screening programmes and lung transplant programmes). We envision the $\mathrm{CF}$ care centre as the patient centred medical home ${ }^{34}$ for the lifelong journey of the individual with CF and his or her family, encompassing the various developmental stages from infancy through adulthood and clinical care for their CF and associated comorbidities.

\section{Deeper patient engagement}

A recent editorial in Health Affairs referred to activated engaged patients as 'the blockbuster drug of the century, ${ }^{35}$ pointing out the association with better outcomes. Indeed, the 'voice of the patient and family' is important in all that we do. At the national level, this includes leadership on the CF Foundation's Board of Trustees, membership on guidelines committees and participation in clinical research processes. At the care centre level, this involves membership on improvement teams and patient/family advisory boards. At the individual level, this includes shared decision making by people with $\mathrm{CF}$ and their families in conjunction with their CF care centre healthcare professionals.

We developed, validated and field tested an experience of care survey ${ }^{36}$ to provide another mechanism for patient and family input on the quality of their care. We have found that care centre level survey results showing strengths and weaknesses compared with results from peer care centres will identify improvement opportunities in service related aspects of care delivery. The infrastructure needed to deploy this survey nationally provides the opportunity to collect additional patient reported outcome measures.

\section{Addressing the treatment burden}

The daily decisions of individuals with CF and their families about their medical regimen impact on clinical outcomes. Clinicians cannot accurately predict which patients are struggling to execute their home based medical regimen and patients are hesitant to report this information. ${ }^{37}$ Opportunities to identify and address challenges are missed. We are developing a patient engagement initiative to (1) identify the obstacles that individuals with CF and families face in successful selfmanagement and (2) equip people with CF, their families and healthcare professionals with the knowledge, skills and resources to overcome these obstacles.

We are also applying comparative effectiveness research methodologies to the patient registry data to assess the impact of specific therapies and combinations of therapies. Given the inherent limitations of observational data, retrospective registry analyses may require validation in prospective trials. We are testing the feasibility of embedding pragmatic, cost effective clinical trials within the registry. ${ }^{38}$

The long term goal is to determine an optimal medical regimen for individuals with CF that is feasible to execute.

\section{Balanced assessment of quality and value of CF care}

We are planning a comprehensive analysis of the cost of care, including direct healthcare expenditures, and indirect costs such as days lost from school or work. Collectively, the clinical outcomes, patient reported outcomes and cost outcomes will provide a broader and more balanced assessment of the quality and value of $\mathrm{CF}$ care. ${ }^{39}$ This information will position the Foundation to better understand what aspects of care and which therapies drive improved outcomes in anticipation of the emerging value based approach to healthcare delivery and payment.

In summary, major advances in basic science and therapeutics development coupled with improvements in healthcare delivery have resulted in striking gains in medical outcomes for people with CF. A devastating illness that took the lives of young children in the past has evolved to a challenging, but manageable, chronic illness. CFTR modulators hold the promise of dramatically extending the length and quality of life for individuals with CF. Incorporation of quality improvement into the CF care model has systematised continuous learning and improvement into a learning health system $^{40}$ to help people with CF and their families, and the CF healthcare professionals that serve them.

Acknowledgements We gratefully acknowledge the leadership and support of Bob Beall and Preston Campbell at the CF

Foundation; the generous sharing of wisdom and experience by Brent James, Don Berwick and Paul Batalden; the too numerous to count contributions of Gerry O'Connor, Hebe Quinton, Margie Godfrey and Kathy Sabadosa at Dartmouth; the dedication of healthcare professionals at CF care centres across the USA; and, most importantly, those whom we serve, people with CF and their families.

Funding The study was funded by the Cystic Fibrosis Foundation. ECN is the principal investigator on a grant (OCONNO04QI1) from the CF Foundation to support the quality improvement initiative.

Contributors Both authors contributed to the conception and design of the work, the analysis and interpretation of the data, and the drafting and revising of the manuscript. Both authors have given approval of the manuscript and take responsibility for its contents.

Competing interests $\mathrm{BCM}$ receives support for salary and professional expenses from the CF Foundation. The CF

Foundation receives a royalty on sales of ivacaftor as a result of its financial support of research that led to its development. The description of ivacaftor within the manuscript is based on medical evidence and not formulated with any intent to affect sales of ivacaftor, because of the Foundation's royalty interest or otherwise.

Provenance and peer review Not commissioned; externally peer reviewed. 


\section{REFERENCES}

1 Andersen DH. Cystic fibrosis of the pancreas and its relation to celiac disease: a clinical and pathologic study. Am J Dis Child 1938;56:344-99.

2 Kessler WR, Andersen DH. Heat prostration in fibrocystic disease of the pancreas and other conditions. Pediatrics 1951;8:648-56.

3 Di Sant'Agnese PA, Darling RC, Perera GA, et al. Abnormal electrolyte composition of sweat in cystic fibrosis of the pancreas; clinical significance and relationship to the disease. Pediatrics 1953;12:549-63.

4 Gibson LE, Cooke RE. A test for concentration of electrolytes in sweat in cystic fibrosis of the pancreas utilizing pilocarpine by iontophoresis. Pediatrics 1959;23:545-9.

5 Quinton PM. Chloride impermeability in cystic fibrosis. Nature 1983;301:421-2.

6 Knowles MR, Stutts MJ, Spock A, et al. Abnormal ion permeation through cystic fibrosis respiratory epithelium. Science 1983;221:1067-70.

7 Riordan JR, Rommens JM, Kerem B, et al. Identification of the cystic fibrosis gene: cloning and characterization of complementary DNA. Science 1989;245:1066-73.

8 Anderson MP, Rich DP, Gregory RJ, et al. Generation of cAMP-activated chloride currents by expression of CFTR. Science 1991;251:679-82.

9 Griesenbach U, Alton EW. Moving forward: cystic fibrosis gene therapy. Hum Mol Genet 2013;22(R1):R52-8.

10 Fuchs HJ, Borowitz DS, Christiansen DH, et al. Effect of aerosolized recombinant human DNase on exacerbations of respiratory symptoms and on pulmonary function in patients with cystic fibrosis. N Engl J Med 1994;331:637-42.

11 Ramsey BW, Pepe MS, Quan JM, et al. Intermittent administration of inhaled tobramycin in patients with cystic fibrosis. N Engl J Med 1999;340:23-30.

12 Saiman L, Marshall BC, Mayer-Hamblett N, et al. Azithromycin in patients with cystic fibrosis chronically infected with Pseudomonas aeruginosa. JAMA 2003;290:1749-56.

13 Elkins MR, Robinson M, Rose BR, et al. A controlled trial of long-term inhaled hypertonic saline in patients with cystic fibrosis. N Engl J Med 2006;354:229-40.

14 Retsch-Bogart GZ, Quittner AL, Gibson RL, et al. Efficacy and safety of inhaled aztreonam lysine for airway pseudomonas in cystic fibrosis. Chest 2009;135:1223-32.

15 McCoy KS, Quittner AL, Oermann CM, et al. Inhaled aztreonam lysine for chronic airway Pseudomonas aeruginosa in cystic fibrosis. Am J Respir Crit Care Med 2008;178: 921-8.

16 Mogayzel PJ Jr, Naureckas ET, Robinson KA, et al. Cystic fibrosis pulmonary guidelines. Chronic medications for maintenance of lung health. Am J Respir Crit Care Med 2013;187:680-9.

17 Flume PA, O'Sullivan BP, Robinson KA, et al. Cystic fibrosis pulmonary guidelines: chronic medications for maintenance of lung health. Am J Respir Crit Care Med 2007;176: 957-69.

18 Trivedi BP. Doorway to a cure. Discover, 2013.

19 Ramsey BW, Davies J, McElvaney NG, et al. A CFTR potentiator in patients with cystic fibrosis and the G551D mutation. N Engl J Med 2011;365:1663-72.

20 Rowe S, Heltshe SL, Gonska T, et al. Results of the G551D observational study: the effect of ivacaftor in G551D patients following FDA approval. Pediatr Pulmonol 2013;278(Suppl 36).
21 Mogayzel PJ, Dunitz J, Marrow LC, et al. Improving chronic care delivery and outcomes: The impact of the Cystic Fibrosis Care Center Network. BMJ Qual Saf 2014;23:i3-8.

22 Cystic Fibrosis Foundation Patient Registry: 2012 Annual Data Report to the Center Directors. Bethesda, Maryland, 2013.

23 Doershuk CF, Matthews LW, Tucker AS, et al. Evaluation of a prophylactic and therapeutic program for patients with cystic fibrosis. Pediatrics 1965;36:675-88.

24 Mantle DJ, Norman AP. Life-table for cystic fibrosis. BMJ 1966;2:1238-41.

25 Moore BM, Laguna TA, Liu M, et al. Increased adherence to CFF practice guidelines for pulmonary medications correlates with improved FEV1. Pediatr Pulmonol 2013;48:747-53.

25 a http://www.cdc.gov/nccdphp/dnpao/growthcharts/resources/ sas.htm.

26 Schechter MS, Fink AK, Homa K, et al. The Cystic Fibrosis Foundation Registry as a tool for use in quality improvement. BMJ Qual Saf 2014;2014;23:19-14.

27 Godfrey MM, Oliver BJ. Accelerating the rate of improvement in cystic fibrosis care: the learning and leadership collaborative. BMJ Qual Saf 2014;23:i23-32.

28 Boyle MP, Sabadosa KA, Quinton HB, et al. Key findings of the Cystic Fibrosis Foundation's US clinical practice benchmarking project. BMJ Qual Saf 2014;23:i15-22.

29 Richards KM, Lester MK, Chin MJ, et al. A preliminary evaluation of the effectiveness of the Cystic Fibrosis Foundation mentoring program for respiratory care. Respir Care 2013;58:764-9.

30 Wenger E. Communities of practice: learning, meaning, and identity. New York: Cambridge University Presss, 1998.

31 Sabadosa KA, Batalden P. The interdependent roles of patients, families and professionals in cystic fibrosis: a system for the co-production of health care and its improvement. BMJ Qual Saf 2014;23:190-94.

32 Nelson EC, Godfrey MM, Batalden PB, et al. Clinical microsystems, part 1 . The building blocks of health systems. Jt Comm J Qual Patient Saf 2008;34:367-78.

33 Moran A, Becker D, Casella SJ, et al. Epidemiology, pathophysiology, and prognostic implications of cystic fibrosis-related diabetes: a technical review. Diabetes Care 2010;33:2677-83.

34 Casalino LP, Rittenhouse DR, Gillies RR, et al. Specialist physician practices as patient-centered medical homes. N Engl J Med 2010;362:1555-8.

35 Dentzer S. Rx for the 'blockbuster drug' of patient engagement. Health Aff (Millwood) 2013;32:202.

36 Homa K, Sabadosa KA, Nelson EC, et al. Development and validation of a cystic fibrosis patient and family member experience of care survey. Qual Manag Health Care 2013;22:100-16.

37 Daniels T, Goodacre L, Sutton C, et al. Accurate assessment of adherence: self-report and clinician report vs electronic monitoring of nebulizers. Chest 2011;140:425-32.

38 Lauer MS, D’Agostino RB Sr. The randomized registry trialthe next disruptive technology in clinical research? N Engl J Med 2013;369:1579-81.

39 Nelson EC, Batalden PB, Godfrey MM, et al. Value by design: developing clinical microsystems to achieve organizational excellence. San Francisco, CA: Jossey-Bass, 2011.

40 Institute of Medicine (IOM). The learning healthcare system: workshop summary. Washington, DC: National Academies Press, 2007. 